\title{
EXCITON TRIONS IN II-VI HETEROSTRUCTURES
}

\author{
R.T. Cox, V. Huard, K. Kheng, S. Lovisa, R.B. Miller, \\ K. SAMINADAYAR
}

CEA-Grenoble, 38054 Grenoble Cedex 9, France

\author{
A. Arnoult, J. Cibert, S. Tatarenko
}

Laboratoire de Spectrométrie Physique, 38402 St Martin D’Hères Cedex, France

AND M. POTEMSKI

Grenoble High Magnetic Field Laboratory, 38042 Grenoble Cedex 9, France

Optical spectra associated with transitions that create or annihilate charged excitons $X^{-}$or $X^{+}$can be observed in quantum well heterostructures containing an electron gas or a hole gas, respectively. A review is given of properties of trion states in CdTe quantum wells in zero field and of the magnetic field-dependence of the circular polarization and oscillator strength of the trion optical resonance. The possibility that disorder is needed to stabilise trion states in concentrated $2 \mathrm{D}$ electron or hole systems is discussed.

PACS numbers: 73.20.Dx, 78.66. Hf, 71.35.Ji, 78.20.Ls

In 1958, M.A. Lampert [1] extended the analogy between the exciton $X$ in a semiconductor and the hydrogen atom $\mathrm{H}$ in free space by postulating the existence of two types of charged exciton species or "trions". The first is $X^{-}$(two electrons bound to one hole). For $m_{\mathrm{h}} \gg m_{\mathrm{e}}$, this is a close analogue of the $\mathrm{H}^{-}$ion where two electrons with antiparallel spins move in a very diffuse orbital around a proton. The second species is $X^{+}$(two holes and one electron). For $m_{\mathrm{h}} \gg m_{\mathrm{e}}$, this is analogous to the $H_{2}^{+}$molecular ion where one electron moves in a molecular orbital around two protons (Lampert suggested the nomenclature $X_{2}^{+}$, but " $X^{+}$" has become more usual).

Both kinds of trion have only a very small binding energy and their observation in bulk semiconductors has proved very elusive. However, the calculated binding energy of trions is enhanced by an order of magnitude in 2 dimensions [2]. Moreover, an excess of electrons (or holes) can readily be produced in a quantum well heterostructure by modulation doping, favoring the formation of $X^{-}$( or $X^{+}$) in the well.

The first clear identification of the trion $X^{-}$was made in 1993 , in the optical spectra of modulation-doped $\mathrm{CdTe}$ quantum wells containing $10^{10}$ to $10^{11}$ electrons $/ \mathrm{cm}^{2}[3]$. Trions $X^{-}[4]$ and $X^{+}$[5] were then identified in GaAs 
quantum wells. More recently, $X^{+}$has been identified in CdTe wells [6], and $X^{-}$ in ZnCdSe [7] quantum wells. II-VI semiconductors are a particularly favorable environment for studying trions because of the large value of the effective Rydberg constant which is an approximate scaling factor for the trion binding energies (for example $R=14 \mathrm{meV}$ in CdTe compared to $7 \mathrm{meV}$ in GaAs).

In this paper, we will review recent work on trions $X^{-}$and $X^{+}$in CdTe quantum wells grown in Grenoble, particularly discussing transmission spectroscopy. Studies of trions and other excitonic effects in modulation-doped CdTe wells grown at University of Würzburg have also been reported by Kochereshko and co-workers [8]. Spectroscopy is now being done in several laboratories on the $n$-type modulation-doped CdTe wells grown at the Institute of Physics, Warsaw, which have the highest mobilities of any CdTe samples grown so far (up to $\left.1.4 \times 10^{5} \mathrm{~cm}^{2} /(\mathrm{V} \mathrm{s})\right)[9]$.

In Grenoble, we grow $\mathrm{CdTe}$ quantum well structures by molecular beam epitaxy on $\mathrm{Cd}_{0.88} \mathrm{Zn}_{0.12} \mathrm{Te}$ substrates which are transparent at the energy of exciton transitions in typical $\mathrm{CdTe}$ quantum wells. Thus, our main experimental technique is the measurement of the optical transmission, which is more quantitative than luminescence or photoluminescence excitation (PLE) measurements. The CdZnTe substrate induces a large compressive strain in a CdTe quantum well and light hole transitions are split off to $35 \mathrm{meV}$ above heavy-hole transitions, even before inclusion of confinement effects. This simplifies the spectra considerably and we study mainly relatively pure heavy-hole exciton states.

The trion $X^{-}$was first investigated in CdTe multiple quantum wells (MQWs) with $\mathrm{Cd}_{1-x} \mathrm{Zn}_{x}$. Te barriers $(x \approx 0.1$ to 0.2 ). More recently, we have been growing structures with $\mathrm{Cd}_{1-x-y} \mathrm{Zn}_{x} \mathrm{Mg}_{y}$ Te barriers. The zinc $(x \approx 0.1)$ is added mainly to lattice match the barrier alloy to the CdZnTe substrate. The magnesium $(x \approx 0.1 \div 0.2)$ increases the barrier band gap but hardly changes the lattice parameter. We produce an electron gas in our CdTe wells by doping the barriers with indium or aluminum donors (the Würzburg and Warsaw laboratories use iodine). Hall measurements of our samples at $2 \mathrm{~K}$ show a (not very well-defined) quantum Hall effect and mobilities $\mu$ of order $10^{4} \mathrm{~cm}^{2} /(\mathrm{V} \mathrm{s})$. A hole gas is obtained in CdTe wells by doping the barriers with nitrogen acceptors ( $\mathrm{Zn}$ in the barriers greatly enhances the $p$-type doping efficiency) [10]. We have also incorporated paramagnetic manganese in some of our structures, in order to study the effects of enhancing the $g$-factor in a $2 \mathrm{D}$ electron or hole system.

Although much has been learnt by growing sets of samples with different carrier concentration $n_{\mathrm{e}}$ or $n_{\mathrm{h}}$, it is very useful to be able to vary the concentration in a given sample. This can be achieved in some samples by optical excitation. A more quantitative method is to insert a single quantum well, modulation-doped on one side only, in a Schottky diode structure [11]. The electron concentration is varied by applying a voltage between the semi-transparent gold Schottky electrode on the surface and a degenerate $n^{+}$-doped layer situated underneath the well. Ideally, $n_{\mathrm{e}}$ is a linear function of $V$, and can be calibrated by measuring the device's capacitance as a function of the applied voltage [12].

Spectroscopy of trions is done at low temperature because trion states disappear at some tens of $\mathrm{K}$, due to their low binding energy. We are interested in 
three absorption/emission transitions:

$$
h \nu_{1} \leftrightarrow X \quad(1 \mathrm{a}), \quad \mathrm{e}+h \nu_{2} \leftrightarrow X^{-} \quad(1 \mathrm{~b}), \quad \mathrm{h}+h \nu_{3} \leftrightarrow X^{+} .
$$

We briefly discuss zero-field spectra before describing the very interesting properties seen in a magnetic field.

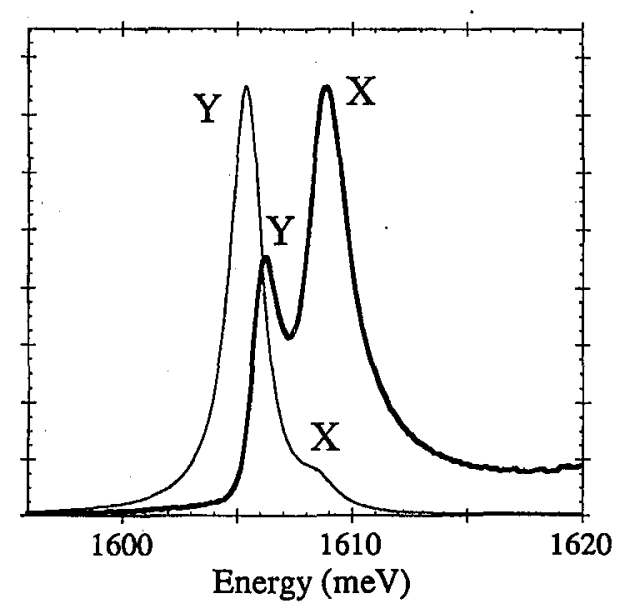

Fig. 1. Optical density (thick trace) and emission (thin trace) spectra at $2 \mathrm{~K}$ for a 10 period $90 \AA \mathrm{CdTe} / \mathrm{Cd}_{0.86} \mathrm{Zn}_{0.16} \mathrm{Te} \mathrm{MQW}$ with $n_{\mathrm{e}} \approx 2 \times 10^{10}$ electrons $/ \mathrm{cm}^{2}$ in each well (sample 2D137). Resolved peaks $X$ and $Y$ correspond to the regular exciton and the negatively charged exciton (trion) $X^{-}$, respectively. The $c a .1 \mathrm{meV}$ "Stokes shift" between emission and absorption is due to interface roughness.

Figure 1 shows absorption and emission for a lightly-doped CdTe MQW ( $n_{\mathrm{e}} \approx 2 \times 10^{10} \mathrm{~cm}^{-2}, 90 \AA$ wells). The peaks labelled $X$ and $Y$ are attributed to the exciton $X$ and to the negatively charged exciton $X^{-}$, respectively. The peak $Y$ is weaker than $X$ in absorption but dominates in emission because of thermalization within the excited states. The $X-Y$ separation gives $E_{\mathrm{b} 2}$, the binding energy of a second electron to an exciton, as can be seen by subtracting Eq. (1b) from Eq. (1a) and remanipulating the result: $X^{-}+\left(h \nu_{1}-h \nu_{2}\right) \leftrightarrow(X+\mathrm{e})$. We measure 2.5 to $3 \mathrm{meV}$ for $E_{\mathrm{b} 2}$ in CdTe QWs of width $L_{\mathrm{w}} \approx 100 \AA$, depending on barrier height, compared to an exciton binding energy $E_{\mathrm{b} 1}$ of $c a .18 \mathrm{meV}$. The value of $E_{\mathrm{b} 2}$ is much enhanced by quantum confinement as compared to the theoretical value of $E_{\mathrm{b} 2}$ in bulk CdTe (about $0.4 \mathrm{meV}$ for $m_{\mathrm{e}} / m_{\mathrm{h}} \approx 0.2$ [2]). But surprisingly, $E_{\mathrm{b} 2}$ is still as high as $1.5 \mathrm{meV}$ for $L_{\mathrm{w}}=600 \AA[13]$, which one might think to be almost a $3 \mathrm{D}$ case. We have not done systematic studies of the well-width dependence.

The positively charged exciton $X^{+}$has been observed in $p$-type modulation doped CdTe QWs of width $L_{\mathrm{w}}=80 \AA[6]$. The binding energy $E_{\mathrm{b} 2}$ (here the binding energy of the second hole) is $2.6 \mathrm{meV}$, as compared to $2.9 \mathrm{meV}$ for $X^{-}$in wells of the same width and barrier height. Theoretical calculations [2] predicted $E_{\mathrm{b} 2}$ to be somewhat higher for $X^{+}$than for $X^{-}$for all mass ratios $m_{\mathrm{e}} / m_{\mathrm{h}}$, but the theory does not consider the real complexity of the valence band. 

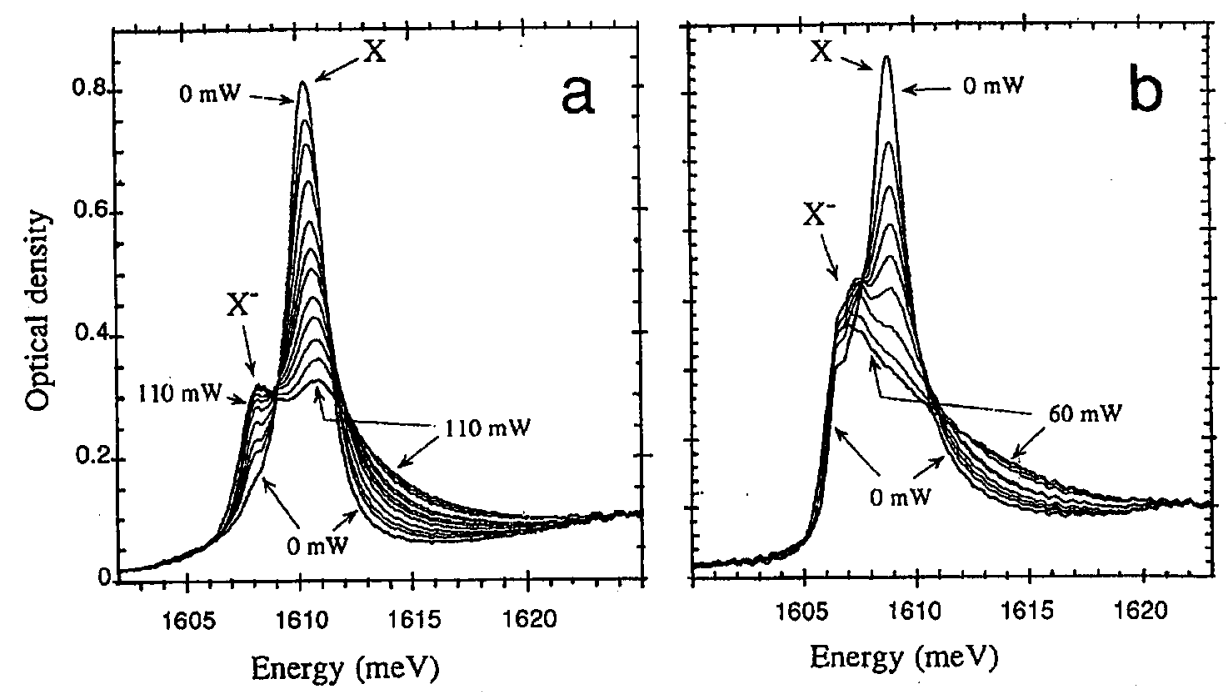

Fig. 2. Optical density (- $\log _{10}$ (transmission)) at $2 \mathrm{~K}$ for two photosensitive $100 \AA \mathrm{CdTe} / \mathrm{CdZnTe} 5$ period MQWs. In sample (a) the initial $2 \mathrm{D}$ electron concentration $n_{\mathrm{e}}$ is insignificant. Under illumination with $1 \mathrm{~mm}$ diameter red laser light beam, electrons accumulate in the CdTe wells, causing emergence of trion peak $X^{-}$with broadening of exciton peak $X$ as laser power increases up to $110 \mathrm{~mW}$. Sample (b) with higher starting $n_{\mathrm{e}}$ gives a higher maximum $n_{\mathrm{e}}\left(\approx 10^{11} \mathrm{~cm}^{-2}\right)$ and shows $X$ and $X^{-}$merging into the beginning of a "Fermi edge enhancement" peak at $60 \mathrm{~mW}$.

Figure 2 shows the evolution of the spectrum as a function of $n_{\mathrm{e}}$ for two $\mathrm{CdTe} / \mathrm{CdZnTe}$ multiple quantum well structures (100 $\AA$ wells) with a special doping that allows us to vary $n_{\mathrm{e}}$ by optical excitation with red laser light [14]. As $n_{\mathrm{e}}$ increases with increasing laser power, the $X$ peak broadens asymmetrically to the high energy side and loses amplitude, due to the effects of phase space filling and of screening and multiple scattering processes. Unfortunately, nearly all the theoretical discussion of these effects (see, e.g., the review by Schmitt-Rink et al. [15]) pre-dated the observation of the trion (1993). To our knowledge Hawrylak was the only theoretician to predict that a two-electron state could contribute to the optical spectrum [16]. Experimentally, for our $100 \AA \mathrm{CdTe} Q W s$, when the absorption peak $X$ broadens and weakens, the trion peak $Y$ appears about $2-3 \mathrm{meV}$ below $X$, see Fig. 2a. The peaks $Y$ and $X$ may be related to "thresholds" $\omega_{1}$ and $\omega_{2}$ of Hawrylak's theory [16,17]. Figure 2b, for a sample giving a higher maximum $n_{\mathrm{e}} \approx 10^{11} \mathrm{~cm}^{-2}$, shows $Y$ and $X$ merging into a single, asymmetric broad peak. This is the beginning of the much-discussed "Fermi edge enhancement" [15].

Figure 2 shows only two possible sets of zero-field spectra. We find rather different line shapes in, for example, the Schottky diode samples. The probable explanation for such differences is disorder, which can be very different in samples having the same electron concentration. In fact, disorder may be very important for stabilising trions in the CdTe samples. Clearly, a hypothetical, disorder-free QW 
containing only a few, widely separated electrons has two well-defined types of excited state, the trion $X^{-}$and the exciton $X$. But from concentrations $10^{10} \mathrm{~cm}^{-2}$, screening and phase-space filling by mobile carriers are in principle very significant unless reduced by disorder-induced localization of the carriers. The donor charges $D^{+}$(concentration $C$ ) in the barriers at a spacer distance $s$ produce very large potential fluctuations in the quantum well plane. These are screened by the electron gas, but in the model of Efros et al. [18], for a critical value of a reduced concentration parameter $\eta=n_{\mathrm{e}} s / \sqrt{C}=0.42$, the screening becomes nonlinear and large islands of dielectric (insulating regions containing no electrons) emerge from a metallic sea (the 2D electron "gas"). Recently, the emergence of the trion peak $Y$ was observed quite precisely at $\eta \approx 0.4$ as $n_{\mathrm{e}}$ was reduced in a Schottky diode sample (S. Lovisa, to be published), which suggests that the trion peak may be associated somehow with the separation of the system into metallic and dielectric regions.

Note that a neutral excitoll very strongly localised by trapping on a single neutral donor very close to or in the QW plane is a quite different species $\left(D^{0} X\right)$ with a hydrogen molecule-like configuration $\left(\mathrm{H}_{2}\right)$. This has been studied [19] in a series of CdTe $100 \AA \mathrm{QW}$ samples doped with donors in the well, or at the well edges, or at distances $s$ progressively further out into the barriers. The binding energy changed from $4.5 \mathrm{meV}$ for $D^{0} X$ to $3 \mathrm{meV}$ for $X^{-}$as $s \rightarrow \infty$ and the oscillator strength "per electron" was ca. 10 times larger for $X^{-}$.

In summary of the zero-field results, CdTe QW spectra show trions and excitons at low electron concentration $n_{\mathrm{e}}$ (or low hole concentration $n_{\mathrm{h}}$ ) but a single broad absorption peak at the Fermi edge is seen at high carrier concentration. A striking property of these samples is that both trion and exciton resonances reappear in the absorption spectra under application of a magnetic field perpendicular to the plane of the well. This is shown in Fig. 3 for a $Q W$ containing $2.2 \times 10^{11}$ electrons $/ \mathrm{cm}^{2}$. Since we have shown field-sweeps for pure CdTe QWs in several previous publications, we present here the spectra of a $100 \AA$ single QW containing $0.2 \% \mathrm{Mn}$, i.e., the well layer is a "very dilute" magnetic semiconductor $\mathrm{Cd}_{0.998} \mathrm{Mn}_{0.002} \mathrm{Te}$. Although complicated to describe, these spectra are instructive because they show very clearly the relation between the spin polarization of the $2 \mathrm{D}$ electron system and the circular polarization of the $X^{-}$absorption.

A strong circular polarization at low temperature is one of the main characteristics of the trion magneto-absorption, for both $X^{-}$and $X^{+}$. For (pure) $\mathrm{CdTe}$ quantum wells at $2 \mathrm{~K}$, the $X^{-}$transition is strongly $\sigma^{+}$polarized and the $X^{+}$transition is strongly $\sigma^{-}$polarized. The reason is that in usual CdTe quantum wells the $g$-factor is negative for a. 2D electron system (unless $\mathrm{Mn}$ is added) and negative for a $2 \mathrm{D}$ hole system. The circular polarization of transition e+photon $\rightarrow X^{-}$is explained in a multi-electron scheme in Fig. 4a. The observed state of the trion $X^{-}$is the "electron spin singlet" state in which its two electrons have antiparallel spins, so the trion has the spin state of the (heavy) hole $m= \pm 3 / 2$. The allowed $(\Delta M= \pm 1)$ transitions in $\sigma^{-}, \sigma^{+}$are then as shown. At low temperature in $\mathrm{CdTe}$, with the electron spins polarized in the spin-up state $m=+1 / 2$, the $\sigma^{+}$ absorption predominates. 


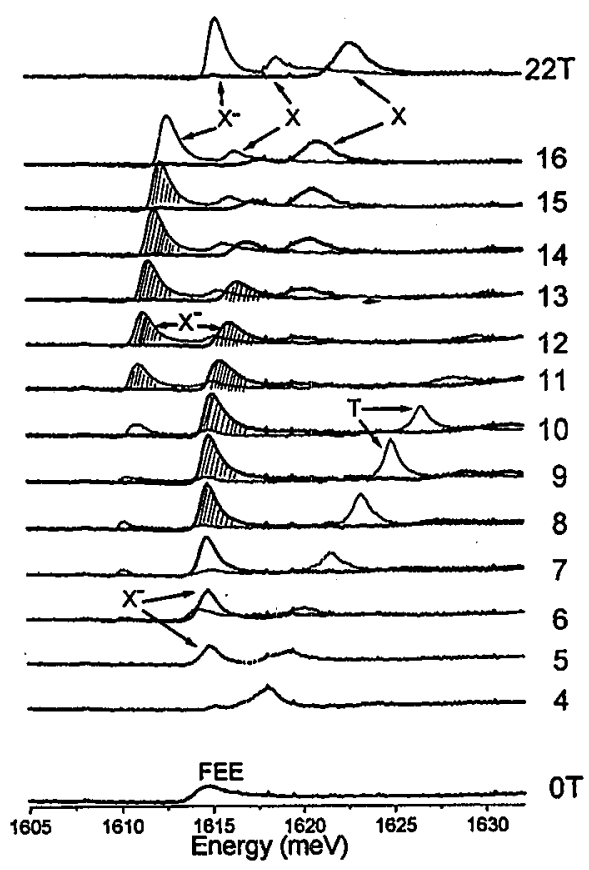

Fig. 3. Absorption spectrum at $2 \mathrm{~K}$ for a $100 \AA \mathrm{CdTe}(0.2 \% \mathrm{Mn}) / \mathrm{CdZnMgTe}$ single quantum well containing $n_{\mathrm{e}}=2.2 \times 10^{11}$ electrons $/ \mathrm{cm}^{2}$ (sample M1020). Magnetic fields $B$ from 0 to $22 \mathrm{~T}$ are applied perpendicular to the QW plane. Thick traces are $\sigma^{-}$ and thin traces are $\sigma^{+}$polarization. Threshold for emergence of trion is $4.5 \mathrm{~T}(\nu=2)$. The $\sigma^{-}$trion peak is strongest at low $B$, where $g_{\mathrm{e}}$ is positive, but the $\sigma^{+}$trion peak predominates after $g_{\mathrm{e}}$ switches negative at $11.8 \mathrm{~T}$.

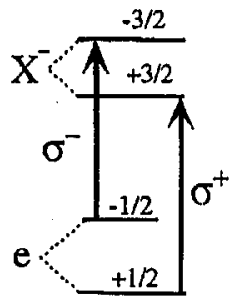

(a)

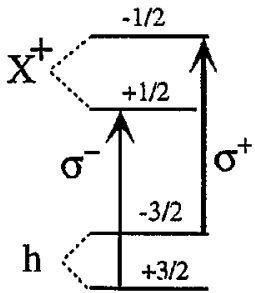

(b)

Fig. 4. Faraday geometry selection rules for transitions that create (a) the trion $X^{-}$ in a CdTe QW containing 2D electrons and (b) the trion $X^{+}$in a CdTe QW containing 2D holes. A magnetic field $B$ is applied perpendicular to the QW plane. At high $B$ and low temperature, the $\sigma^{+}$transition dominates in (a) and the $\sigma^{-}$transition dominates in (b).

In $X^{+}$; the two holes spins are antiparallel, so $X^{+}$has the spin $m= \pm 1 / 2$ of its single electron, see Fig. $4 \mathrm{~b}$. With $g_{\mathrm{h}}$ negative in $\mathrm{CdTe}$, the $2 \mathrm{D}$ hole system is 
polarized $m=+3 / 2$, so the absorption transition $\mathrm{h}+$ photon $\rightarrow X^{+}$is $\sigma^{-}$polarized. This helps to distinguish $X^{+}$and $X^{-}$transitions, which lie very close together in CdTe [6]. The circular polarization characteristic also helped to identify $X^{-}$ transitions in $\mathrm{ZnCdSe}$ [7] quantum wells: there $g_{\mathrm{e}}$ is positive and $X^{-}$has $\sigma^{-}$ polarization in absorption and reflectivity.

In the $\mathrm{Mn}$-doped sample of Fig. 3 at $2 \mathrm{~K}$, due to exchange interaction with the polarized $\mathrm{Mn}$ spins, there is a large splitting of the electron spin levels at very low $B$, giving a positive sign to the Lande $g$-factor $g_{\mathrm{e}}$. This Mn-related "giant" splitting quickly saturates with $B$. The normal linear Zeeman effect of CdTe has negative sign $\left(g_{\mathrm{CdTe}}=-1.6\right)$ and so, from $c a .2 \mathrm{~T}$, the electronic Zeeman splitting decreases almost linearly with field. The effective $g$-factor passes through zero at $12 \mathrm{~T}$ and then becomes negative as in pure CdTe QWs.

In zero-field, the absorption spectrum of this sample consists of a broad Fermi edge enhancement (bottom trace in Fig. 3). This peak splits into two branches polarized $\sigma^{-}$(upper branch) and $\sigma^{+}$(lower branch) in very small $B$, with $80 \%$ of the splitting in the valence band. The Mn-related splitting saturates at $4 \mathrm{meV}$ and the two branches resolve into peaks appearing at successively smaller integer values $(8,7, \ldots 3)$ of the Landau level filling factor $\nu=n_{\mathrm{e}} h / e B$ (region $0-4 \mathrm{~T}$, not shown in Fig. 3). These are essentially unbound electron-hole excitations, from the valence band Landau levels to the emptying conduction band Landau levels $n=3,2,1$ and they move approximately linearly with $B$. Such transitions have also been seen in PLE spectroscopy by Shen for $n$-type modulation doped CdTe and CdMnTe QWs grown at Würzburg [20].

At filling factor $\nu=2(4.5 \mathrm{~T})$, the trion absorption peak $X^{-}$appears and then grows rapidly in intensity with increasing $B$ (decreasing $\nu$ ), see Fig. 3 . It is identified by its quadratic energy dependence on field - a key characteristic of exciton states - and, in this CdTe:Mn sample, its initial $\sigma^{-}$polarization (instead of $\sigma^{+}$in pure CdTe). That the trion $X^{-}$emerges systematically at $\nu=2$ (as seen already in Ref. [3]) has been confirmed recently by field-sweeps of a diode sample at various $n_{\mathrm{e}}$ values. The $\nu=2$ threshold can be used to deduce $n_{\mathrm{e}}$ when $n_{\mathrm{e}}$ is unknown, except at very low $n_{\mathrm{e}}$, where a trion-like absorption peak $Y$ can often be seen in zero-field as noted above.

The intensity variations seen in Fig. 3 for our $\mathrm{CdTe}(0.2 \% \mathrm{Mn}) \mathrm{QW}$ provide a striking illustration of the trion selection rules. From its emergence at $4.5 \mathrm{~T}$ $(\nu=2)$ up to $12 \mathrm{~T}$, the $\sigma^{-}$trion absorption peak dominates, because $g_{\mathrm{e}}$ is positive in this field range and it is the $m=-1 / 2$ state that is populated. But the $\sigma^{+}$ trion emerges ( $4 \mathrm{meV}$ lower due to the Mn-exchange splitting) as $g_{\mathrm{e}}$ decreases and, from $11.8 \mathrm{~T}$, where $g_{\mathrm{e}}$ goes through zero, becomes predominant as in pure $\mathrm{CdTe}$. At $22 \mathrm{~T}$, the $\sigma^{-}$trion has disappeared completely.

We now discuss the filling factor dependence of the trion's oscillator strength. We have measured $f$, the absolute oscillator strength per square $\mathrm{cm}$ of the $X^{-}$. absorption peak in $\sigma^{+}$polarization, at $2 \mathrm{~K}$ as a function of $n_{\mathrm{e}}$ at various fixed fields $B$. The sample was a $100 \AA$ (pure) CdTe well inserted in a Schottky diode [11]. Idealised, the graph obtained for $f$ is an equilateral triangle peaking at $\nu=1$. That is, starting from $n_{\mathrm{e}}=0$, the oscillator strength $f$ increases along a field-independent straight line up to $\nu=1$, then decreases with the same slope to disappear at the 


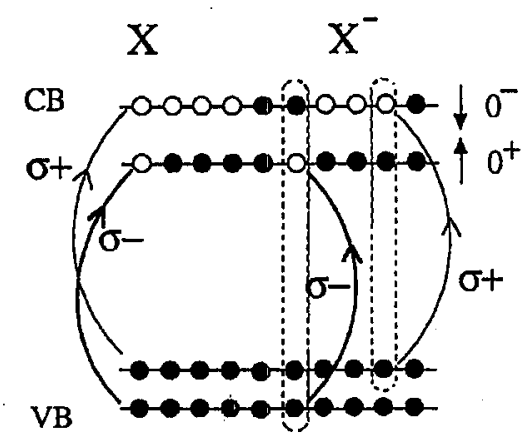

Fig. 5. Allowed absorption transitions from the valence band to the lowest conduction band Landau levels $0^{+}$and $0^{-}$, for polarizations $\sigma^{+}$(thin arrows) and $\sigma^{-}$(thick arrows). Black circles (electrons) and open circles (holes) illustrate a situation with $1<\nu<2$ at a low but finite temperature that gives some holes in the $0^{+}$level. In a high field model of excitonic states, the transitions on the right create trions $X^{-}$(2 conduction electrons bound to one valence hole) and the transitions on the left create excitons $X$.

$\nu=2$ threshold. This "triangular" variation is cleanest at the highest field $(8 \mathrm{~T})$. It is less well-defined at low $B$ where the Landau levels are not so well resolved.

Such a variation is understandable in a "high-field" model of the trion shown in a one-electron scheme in Fig. 5 , where only the $n=0$ conduction and valence band Landau levels are considered. The conduction band Landau level has two sublevels $0^{+}(\operatorname{spin} m=+1 / 2)$ and $0^{-}(m=-1 / 2)$. The oscillator strength of any $\sigma^{+}$transition from the valence band terminating on the $0^{-}$conduction band sublevel is proportional to the number of "holes" $n_{\mathrm{h}}\left(0^{-}\right)$in this $0^{-}$sublevel, or in other words to the number of unoccupied spin-down $n=0$ states. For the transition that creates a trion $X^{-}$, this number has to be multiplied by the probability that the $n=0$ destination state already contains a spin-up electron. This probability is $n_{\mathrm{e}}\left(0^{+}\right) / d$, where $n_{\mathrm{e}}\left(0^{+}\right)$is the number of $m=+1 / 2$ electrons and $d$ is the degeneracy $e B / h$ of a Landau (sub)level. That is

$$
f\left(\sigma^{+}\right)=f n_{\mathrm{h}}\left(0^{-}\right) n_{\mathrm{e}}\left(0^{+}\right) / d,
$$

where $f\left(\sigma^{+}\right)$is in $\mathrm{cm}^{-2}$ and the constant $f$ is a dimensionless number. For $1<\nu<2$ at low $T$, high $B$, the term $n_{\mathrm{e}}\left(0^{+}\right) / d \approx 1$ and the oscillator strength is just given by the number of empty states. For $\nu<1, n_{\mathrm{h}}\left(0^{-}\right) / d \approx 1$ in Eq. (2), then $f\left(\sigma^{+}\right)$is determined by the number of electrons and the quantity $f$ can be interpreted as the oscillator strength per electron. Formula (2) then fits the $8 \mathrm{~T}$, $2 \mathrm{~K}$ "triangular" graph very well and we extract $f=70$ for a $100 \AA \mathrm{CdTe} \mathrm{QW}$. The formula is generalisable thus

$$
f\left(\sigma^{ \pm}\right)=f n_{\mathrm{h}}\left(0^{\mp}\right) n_{\mathrm{e}}\left(0^{ \pm}\right) / d .
$$

The validity of formula (2) at $8 \mathrm{~T}$ is nevertheless surprising, because Fig. 5 is not really applicable at $8 \mathrm{~T}$ in CdTe (the ratio of interband Landau energy to exciton binding energy $\gamma \approx 0.35$ only). Rather, one should think of the trion at $8 \mathrm{~T}$ as an inner excitonic "core" (a mixture of different Landau orbitals) binding a second 
electron which occupies a $n=0$ Landau orbital. But we have no theory better than this simple model.

We now discuss the "regular" (neutral) exciton $X$, which appears at higher field than the trion $X^{-}$. In clearcut cases (for example [3]), as we sweep $B$ up, $X$ appears close to $\nu=1$, first in $\sigma^{-}$, somewhat later in $\sigma^{+}$in (pure) CdTe. In Fig. 3, with $g_{\mathrm{e}}$ inverted initially, $X$ appears around $9-10 \mathrm{~T}$ in $\sigma^{+}$, but the $\sigma^{-}$exciton is indeed strongest at high fields.

From Fig. 5, one can infer a $\nu=1$ threshold for $X$, but for both the $\sigma^{-}$and $\sigma^{+}$excitons. We assume, as for $X^{-}$, that the absorption transition $X$ in $\sigma^{+}\left(\sigma^{-}\right)$ polarization is proportional to the number of empty spin-down (spin-up) $n=0$ destination states. We assume as well that exciton formation is possible only if the destination $n=0$ state also contains no spin-up (spin-down) electron, as shown at left in Fig. 5; otherwise the absorbed photons can form only trions. As $B$ is swept up, completely empty $n=0$ Landau levels become available in large numbers from $\nu=1$, allowing both $\sigma^{+}$and $\sigma^{-}$excitons.

Since experimentally $X$ always appears rather later in $\sigma^{+}$(in pure $n$-CdTe), giving the $X$ peak a contrary polarization to the $X^{-}$peak (see $22 \mathrm{~T}$ in Fig. 3 ), the availability of empty states cannot be the only factor. Useful information came from measurements at $2 \mathrm{~K}$ of the relation between the integrated intensity $I$ of the exciton and trion absorption peaks as $n_{\mathrm{e}}$ varied (in the range $\nu<1$ ) at a fixed field of $8 \mathrm{~T}$ [14]. In $\sigma^{+}$polarization, the data fit the following polynomial:

$$
I(X)=I_{0}(X)-a I\left(X^{-}\right)-c I\left(X^{-}\right)^{3},
$$

where $I_{0}(X)$ is the exciton's intensity at $n_{\mathrm{e}}=0$. A value $a \approx 0.9$ was found, very close to unity, with no significant quadratic term for 3 samples studied. The linear term in Eq. (4) represents the "intensity sharing" effect proposed in the previous paragraph ( $X$ transitions become $X^{-}$transitions in a 1:1 correspondence as the $n=0$ Landau level fills). From formula (2), $I\left(X^{-}\right)$is a scale of $n_{\mathrm{e}}$ (for $2 \mathrm{~K}, \nu<1$ ), so the third term in (4) is cubic in $n_{\mathrm{e}}$. Since the peak $X$ was Lorentzian-shaped and broadened strongly as $n_{\mathrm{e}}$ increased, the term in $n_{\mathrm{e}}^{3}$ was attributed to effects of screening and scattering on $X$. These can be very different in different samples, explaining the variability of $I(X)$ at a given $\nu$.

There is no space here to discuss the very interesting line labelled $T$ in Fig. 3, which appears in the contrary polarization to $X^{-}$over a broad region $2>\nu>0$ and moves approximately linearly with $B$. We attributed line $T$ to an excited "electron spin triplet" state of $X^{-}$, with one electron on the $n=0$ Landau level and the second electron with parallel spin on the $n=1$ level [21]. Observing the same line at lower $n_{\epsilon}$, Kochereshko and co-workers have interpreted it rather differently as a creation of an exciton $X$ with simultaneous shakeup of a background electron to the $n=1$ level. [22], and they supported their interpretation with a quantitative theory of the momentum conservation requirements.

To conclude, perhaps the most important unanswered question about trions is this: why are trions $X^{-}$not identified in absorption spectra of very low-disorder GaAs/GaAlAs structures? We refer particularly to the extensive transmission studies by Goldberg on wide, one-side modulation doped GaAs QWs with $\mu>10^{6} \mathrm{~cm}^{2} /(\mathrm{V} \mathrm{s})$ [23]. In their swept field experiments, the Boston workers see a $\sigma^{+}$polarized absorption transition to the $n=0$ conduction level appearing 
from $\nu=2$, and a $\sigma^{-}$transition from $\nu=1$. They consider that these create unbound electrons and holes: in their view, excitonic states are totally destabilized by the mobile electrons and do not exist. Thus their model of the absorption has only two resonances (these $\sigma^{+}$and $\sigma^{-}$unbound carrier transitions) where our model (Fig. 5) with its distinguishable trion and exciton states has four ( $X$ and $X^{-}$in $\sigma^{+}$and $\sigma^{-}$).

To reconcile these two models one must presumably incorporate the very different degree of disorder that gives $\mu \approx 10^{6}$ and $10^{4} \mathrm{~cm}^{2} /(\mathrm{V} \mathrm{s})$, respectively in the GaAs and CdTe QWs. But it is interesting that spectra of the CdTe QWs grown in Warsaw are turning out similar to those of the Grenoble samples, even though they have greatly improved mobility $\left(\approx 10^{5} \mathrm{~cm}^{2} /(\mathrm{V} \mathrm{s})[9]\right)$. As noted earlier, strong potential fluctuations can localise the $2 \mathrm{D}$ electrons, reducing the screening and phase-space filling effects that destabilize exciton states. In extreme cases (very low $n_{e}$ ), one could have one electron per fluctuation or less. Nevertheless, because the average length of the potential fluctuations is at least $c a .5$ times the spacer distance $s$ (see [18]), there have to be several electrons per fluctuation for medium-doped samples like that of Fig. $3\left(n_{\mathrm{e}}=2.2 \times 10^{11} \mathrm{~cm}^{-2}, s=250 \AA\right)$. Finally, the theoretical problem of these CdTe systems may be equivalent to that of a set of multiple occupied quantum dots. A better experimental understanding probably requires spatially resolved microspectroscopy or nanospectroscopy, scanning across the quantum well plane.

Many other Grenoble colleagues, including T. Baron, F. Bassani, A. Haury, N. Magnea, Y. Merle d'Aubigné, have also contributed to the work reviewed in this paper.

\section{References}

[1] M.A. Lampert, Phys. Rev. Lett. 1, 450 (1958).

[2] B. Stébé, A. Ainane, Superlattices Microstruct. 5, 545 (1989).

[3] K. Kheng, R.T. Cox, Y. Merle d'Aubigné, K. Saminadayar, S. Tatarenko, Phys. Rev. Lett. 71, 1752 (1993).

[4] G. Finkelstein, H. Shtrikman, I. Bar-Joseph, Phys. Rev. Lett. 74, 976 (1995).

[5] A.J. Shields, J.L. Osborne, M.Y. Simmons, M. Pepper, D.A. Ritchie, Phys. Rev. $B$ 52, 5523 (1995); G. Finkelstein, H. Shtrikman, I. Bar-Joseph, Phys. Rev. B 53, 1709 (1995).

[6] A. Haury, A. Arnoult, V.A. Chitta, J. Cibert, Y. Merle d'Aubigné, S. Tatarenko, A. Wasiela, Superlattices Microstruct. 23, 1097 (1998).

[7] K. Kheng, R.T. Cox, V.P. Kochereshko, K. Saminadayar, S. Tatarenko, F. Bassani, A. Franciosi, Superlattices Microstruct. 15, 253 (1994).

[8] D.R. Yakovlev, V.P. Kochereshko, R.A. Suris, W. Ossau, A. Waag, G. Landwehr, P.C.M. Christianen, J.C. Maan, in: Proc. 23rd Int. Conf. Phys. Semiconductors, Berlin 1996, Eds. M. Scheffler, R. Zimmermann, World Scientific, Singapore 1996, p. 2071 and J. Cryst. Growth 184/185, 818 (1998).

[9] G. Karczewski, J. Jaroszyński, A. Barez, M. Kutrowski, T. Wojtowicz, J. Kossut, J. Cryst. Growth 184/185, 814 (1998).

[10] T. Baron, K. Saminadayar, N. Magnea, J. Appl. Phys. 83, 1354 (1998). 
[11] S. Lovisa, R.T. Cox, N. Magnea, K. Saminadayar, Phys. Rev. B 56, 12787 (1997).

[12] S. Lovisa, R.T. Cox, N. Magnea, K. Saminadayar, J. Cryst. Growth 184/185, 810 (1998).

[13] K. Kheng, K. Saminadayar, N. Magnea, J. Cryst. Growth 184/185, 849 (1998).

[14] R.B. Miller, T. Baron, R.T. Cox, K. Saminadayar, J. Cryst. Growth 184/185, 823 (1998).

[15] S. Schmitt-Rink, D.S. Chemla, D.A.B. Miller, Adv. Phys. 38, 89 (1989).

[16] P. Hawrylak, Phys. Rev. B 44, 3824 (1991).

[17] S.A. Brown, J.F. Young, J.A. Brum, P. Hawrylak, Z. Wasilewski, Phys. Rev. B 54, 11082 (1996).

[18] A.L. Efros, F.G. Pikus, V.G. Burnett, Phys. Rev. B 47, 2233 (1993).

[19] K. Kheng, R.T. Cox, M. Mamor, K. Saminadayar, S. Tatarenko, J. Phys. IV (France) 3, C5, 95 (1993).

[20] J.X. Shen, W. Ossau, F. Fisher, A. Waag, G. Landwehr, Surf. Sci. 361/362, 460 (1996) and Acta Phys. Pol. A 88, 1033 (1995).

[21] R.T. Cox, K. Kheng, K. Saminadayar, T. Baron, S. Tatarenko, in: High Magnetic Fields in the Physics of Semiconductors, World Scientific, Singapore 1995, p. 394.

[22] D. Yakovlev, V.P. Kochereshko, R.A. Suris, H. Schenk, W. Ossau, A. Waag, G. Landwehr, P.C.M. Christianen, J.C. Maan, Phys. Rev. Lett. 79, 3974 (1997).

[23] For example, E.H. Aifer, B.B. Goldberg, D.A. Broido, Phys. Rev. Lett. 76, 680 (1996); B.B. Goldberg, D. Heiman, A. Pinczuk, Phys. Rev. Lett. 63, 1102 (1989). 